\title{
PRODUCTION AND AVAILABILITY OF RADIOISOTOPES
}

\author{
By PAUL C. AEBERSOLD
}

(From the Isotopes Division, U. S. Atomic Energy Commission, Oak Ridge, Tenn.)

Radioisotopes as tracer atoms and as uniquely applicable sources of radiation have become important working tools of clinical investigation. Indeed, they have already been established as permanent tools, indispensable to many investigations. They provide a new and dynamic power of perception-an ultra-sensitive way of following certain atoms. Their application has led to the development of new and unique techniques for studying the metabolism of a wide variety of elements and biochemical compounds, diagnosing and treating disease and studying the behavior and role of pharmaceuticals and other medicinal agents. However, the greatest value of isotopes is expected to be derived from their contribution to the over-all advance of medical knowledge.

\section{PRODUCTION}

The uranium chain-reacting pile has two unique characteristics, the production of a fission chain reaction and the production of vast quantities of neutrons. Both of these features make the reactor a useful and extremely productive unit for making radioisotopes; the first for yielding the so-called "fission products," and the second for producing artificially induced radioactivity. It is the second feature, however, which permits the production of the greater variety of radioelements and the production of isotopes of elements generally found in biological material.

Even before the last war and before the advent of atomic energy reactors, radioisotopes were, of course, producible with high energy "atom smashing" devices, such as the cyclotron. Indeed, investigators using cyclotron-produced isotopes had found in them the most sensitive tool ever placed at their disposal. They also found the radioisotope to be a tool which will permit tracing a specific batch of atoms through a complicated physical, chemical or biological system even though the radioactive atoms may become mixed with many other stable atoms of the same element.

There was one drawback, however, to their widespread utilization at that time. Cyclotron production of radioisotopes was and still is both limited and costly. Thus at the start of the war, science had in radioisotopes a research tool guaranteeing high sensitivity and high specificitya tool in great demand but in small availability.

During the wartime development of the atomic energy project it became evident to scientists who worked with the chain-reacting uranium pile that it would be an excellent unit for producing huge quantities of radioactivity or radioisotopes-for making these valuable tools available on a very wide scale. Out of this realization grew the program for the distribution of pile-produced radioisotopes which was initiated by the Manhattan Project and further developed by the Atomic Energy Commission.

As indicated earlier, radioactive isotopes are created in chain-reacting piles by two processes (1) fission of uranium 235 or other fissionable nuclei and (2) neutron absorption by non-fissionable nuclei placed in the pile for the purpose.

\section{Fission Process}

The fission process results generally in two radioactive fragments, one to three neutrons and several gamma rays as indicated in Figure 1. The total mass of the fragments plus the mass of the one to three neutrons which are emitted during the fission event is equal to the mass of the U 235 nucleus (except for the loss of about onetenth of 1 per cent of the mass which is converted into energy and which causes the tremendous release of energy during fission). The fission fragments are very unstable nuclear species ranging from element number 30, zinc, to element number 64, gadolinium. Each of these species approaches a stable nuclear state by a series or chain of beta ray emissions. The intermediate decay products formed during the beta chain decay are the fission products.

These fission products, because they differ chemically from the original uranium and other possible transuranic contaminants, may be isolated in groups or as isotopes of a single element. The only radioisotope of biological significance produced in this way, however, is radioiodine, $\mathrm{I}^{131}$. 


\section{URANIUM FISSION AND BETA CHAIN DECAY}
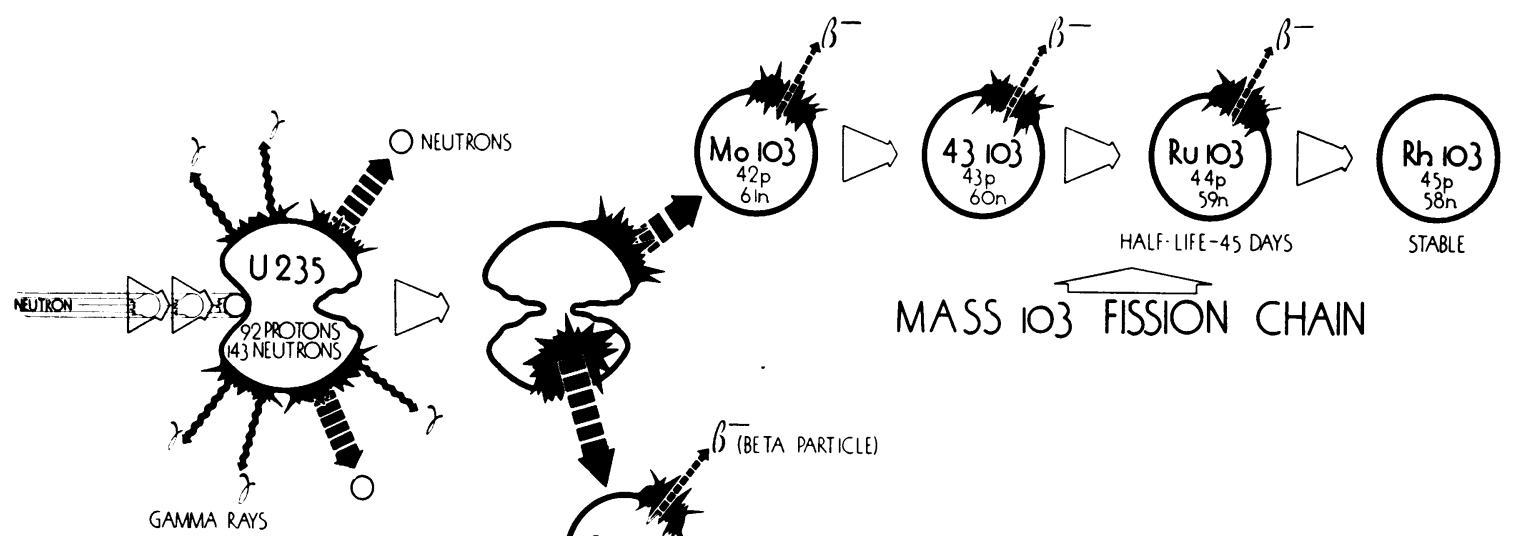

"RADIOIODINE I3I-
A PRODUCT OF THIS CHAIN"

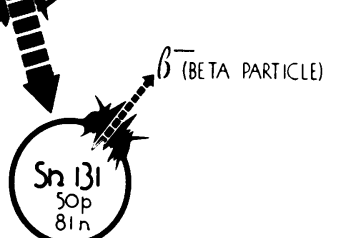

\

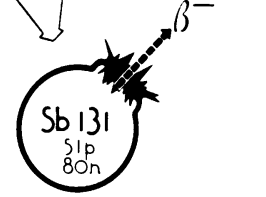

$\triangle M A S S$ I3I FISSION CHAIN

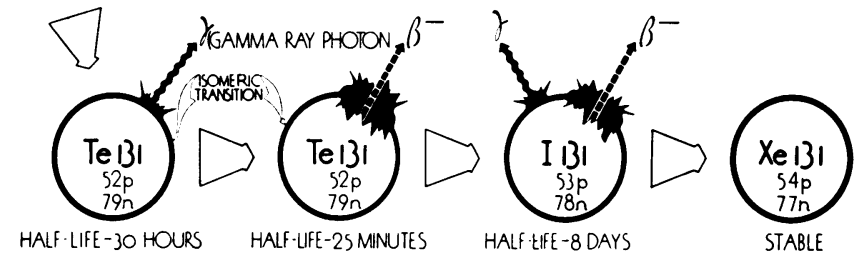

Fig. 1. Uranium Fission Chain Reaction

When Uranium 235 fissions it splits into two fragments which may range in weight from 65 to 157 or from element number 30 , zinc, to element number 64 , gadolinium. On the basis of probability, however, a greater percentage of the fragments range from element number 38 to 45 , and from number 54 to 61 . Each of the radioactive fragments approaches a stable nuclear state by a series or chain of beta ray emissions. The intermediates formed during this decay are the source of the fission products, such as radioiodine 131. Although the above illustration shows only one of many ways in which a U 235 atom may fission, it was chosen as a single example in which a conservation of mass and number of nuclear particles can be shown. It is also an excellent example of how a biologically useful radioisotope, iodine 131 , may be produced through uranium fission.

The production of radioiodine as a fission product is shown in Figure 1.

\section{Neutron Activation}

The pile production process which yields radioisotopes of a much wider variety of elements, in fact, radioisotopes of most of the elements from hydrogen to plutonium, is neutron absorption. Generally, however, the neutron absorption process is subdivided into two categories: (1) simple neutron capture in which only a gamma ray photon is emitted and the product is hence an isotope of the target element and (2) transmutation in which a proton or alpha particle is emitted and the product is hence an isotope of a different element than the target.

The neutron absorption process of making radioisotopes in the pile is extremely prolific because of the pile's capacity to produce tremendous quantities of neutrons. Indeed, neutrons can be produced greatly in excess of those needed to sustain the chain reaction, and these excess neutrons are available for other uses, such as the bombardment of stable materials to form radioactive materials. In the pile at the Oak Ridge National Laboratory where most of the radioisotopes distributed by 
the Atomic Energy Commission are prepared, the "flux" or density of the "atmosphere" of neutrons inside the pile is approximately $10^{12}$ (a million million) neutrons flowing through a square centimeter each second. This value seems even more impressive when one considers that this density exists in a volume occupied by many tons of graphite and uranium.

For the details of production of radioisotopes by neutron absorption, attention is called to Figure 2. As indicated in the illustration, ordinary elements like carbon and phosphorus may be changed

\section{PILE PRODUCTION OF RADIOISOTOPES}

\section{NEUTRON CAPTURE}
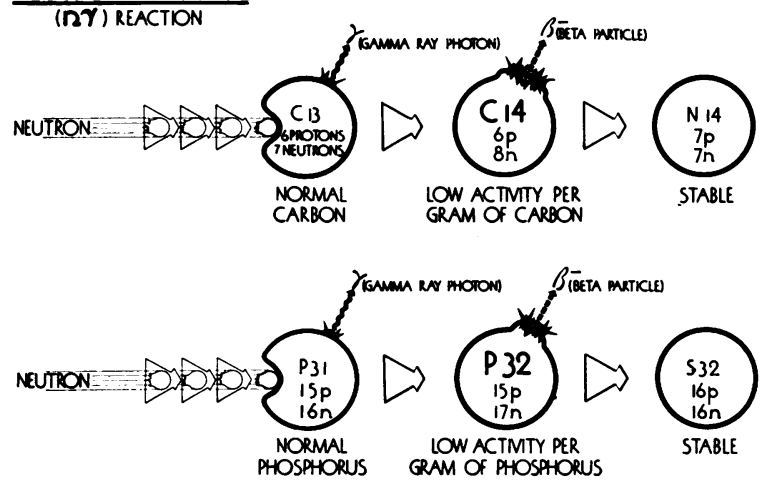

\section{TRANSMUTATION}

(n P) REACTION
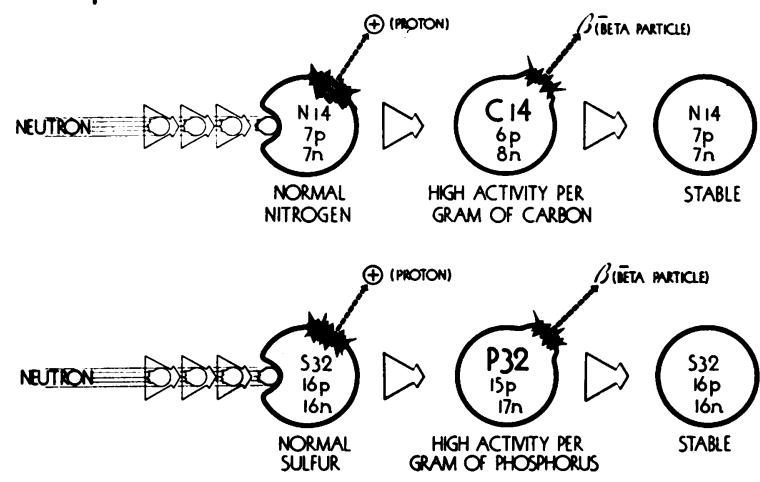

Fig. 2. Pile Production of Radioisotopes

There are two ways of producing radioisotopes by neutron absorption in a nuclear reactor. The first, neutron capture, is effected by the capture of a neutron and the emission of a gamma ray photon yielding a radioisotope of the same element as the target material. The second, transmutation, is effected by the capture of a neutron and the emission of a proton or alpha particle yielding a radioisotope non-isotopic with the target element. Radiophosphorus 32 and radiocarbon 14 may be produced by either or both of these reactions. into their radioactive counterparts by the absorption of a neutron and the emission of a photon of gamma radiation. This reaction, usually referred to as an ( $n$, gamma) reaction, is by far the most prevalent production reaction used in radioisotope preparation. It is not, however, the most practical in all cases. For instance, in the production of radioactive carbon 14 by the ( $n$, gamma) reaction, a neutron must be absorbed by the stable isotope, carbon 13. Although carbon 13 is found in all naturally occurring carbon, it has a normal abundance of only 1.1 per cent. To obtain an efficient yield of carbon 14 by this method would therefore necessitate bombarding a carbon target whose carbon 13 content had been concentrated many times above this abundance. This procedure would, however, prove time-consuming and expensive. Furthermore, it is even less feasible in light of the fact that the carbon 13 isotope has an extremely low probability for capture of "slow" or thermal energy neutrons (low neutron capture cross section). With radioactive phosphorus 32, however, the situation is quite different, in fact the ( $n$, gamma) reaction is very practical for its production. Because naturally occurring phosphorus has only one stable isotope (phosphorus 31 ) and because this isotope has a high probability for capturing thermal energy neutrons, a good yield of radioactive phosphorus results from the ( $n$, gamma) reaction.

The radioisotope formed by an ( $\mathrm{n}$, gamma) reaction is merely the radioactive counterpart of the stable element used as the target material. The radioisotope in this case, therefore, will always be diluted with the stable atoms of the target element which have not been made radioactive during the pile irradiation. Because both the radioactive and stable atoms are of the same element, no chemical separation can be effected. The resultant activity per gram of element is called the "specific activity." It is dependent on the chance for capture of a neutron (capture cross section) by the target nucleus, the neutron "flux" of the production pile and the duration of the irradiation. The specific activity of a radioisotope may be extremely important for its use as a tracer because it determines to what extent the radioisotope may be diluted and still afford reliable detection and measurement. 
On the other hand, a radioisotope produced by a transmutation reaction is an isotope of a different element than the target element (non-isotopic with the target element). It may, therefore, be chemically separated from the residual stable atoms in the target after pile irradiation. A radioisotope produced in this way and then chemically separated from the irradiated material, i.e., from the stable isotopes of the same element, is called "carrier free." In practice some "carrier" or stable element is usually added to facilitate radiochemical handling. Nevertheless, radioisotopes produced by transmutation have a much higher specific activity than those produced by an (n, gamma) reaction. For this reason, transmutation is a particularly useful production reaction. The chemically extracted transmuted radioisotope will be diluted only by stable element present as impurity in the compound irradiated, or by stable element added as carrier to help extract the radioisotope. It can thus undergo great dilution in a tracer experiment before becoming undetectable.

As also indicated in Figure 2, radiocarbon 14 and radiophosphorus 32 may be produced by a transmutation reaction. In both cases a neutron is absorbed and a proton is emitted during the reaction. In the case of carbon 14 production, the target material is a nitrogen compound (calcium nitrate) while for phosphorus 32 the target material is elemental sulfur. Both of these target nuclei have a high natural abundance in the element and both target materials are readily available and may be obtained quite pure chemically.

There is still another type of transmutation reaction used in the pile production of radioisotopes-the reaction in which a neutron is absorbed and an alpha particle is emitted. This reaction, however, is feasible for the production of only two isotopes, radiohydrogen ( $\mathrm{H} 3$ or tritium) from lithium 6 and radioargon (A 37) from calcium 40 .

The mechanical operations associated with pile production of radioisotopes is quite simple. Reference is made to Figure 3 showing a schematic diagram of the pile at the Oak Ridge National Laboratory which is used to make most of the radioisotopes now being distributed by the Commission. In the case of the fission products, the radioisotopes are chemically extracted from the active uranium slugs which have previously been discharged into a channel of water at the rear of the pile.

If the radioisotope has resulted from an ( $n$, gamma) reaction, it is usually shipped as an irradiated unit. This means that the material in the aluminum test tube, consisting of stable atoms of the target material together with their counterparts which have been made radioactive, is merely removed and packaged prior to shipping. If, on the other hand, the radioisotope has been produced by a transmutation reaction, it may be chemically separated from the target material prior to packaging and shipping.

\section{Production Status}

When the radioisotope distribution program was initiated, production scheduling was based primarily on the expected demand of medical investigators. To a great extent the same basis is used in scheduling current production. At least two reasons account for this situation. In the first place a majority of potential radioisotope users were and still are associated with some phase of medical or biological research. In the two and a half year period since the inauguration of the distribution program, nearly 75 per cent of the radioisotope shipments have been used for studies in medical therapy, animal physiology or bacteriology.

The second reason for special effort in preparing the radioisotopes needed by medical research is associated with the characteristics of the isotopes generally used in these investigations. Many of the biologically useful radioisotopes are short lived materials, that is, over a comparitively short period of time most of the radioactivity of the isotope will have disappeared through spontaneous disintegration. Indeed, if the radioisotope is to be used in human studies, it is desirable that it have a short life of radioactivity in order to prevent extended radiation exposure of the patient. It is difficult, however, to supply materials of short half-life because they cannot be stockpiled for an appreciable length of time. They must be produced currently to meet current and fluctuating demands. Of the total number of radioisotope shipments made thus far over 65 per cent have been shipments of phosphorus 32 or iodine 131, having respective half-lives of 14.3 days and 8.0 days. 


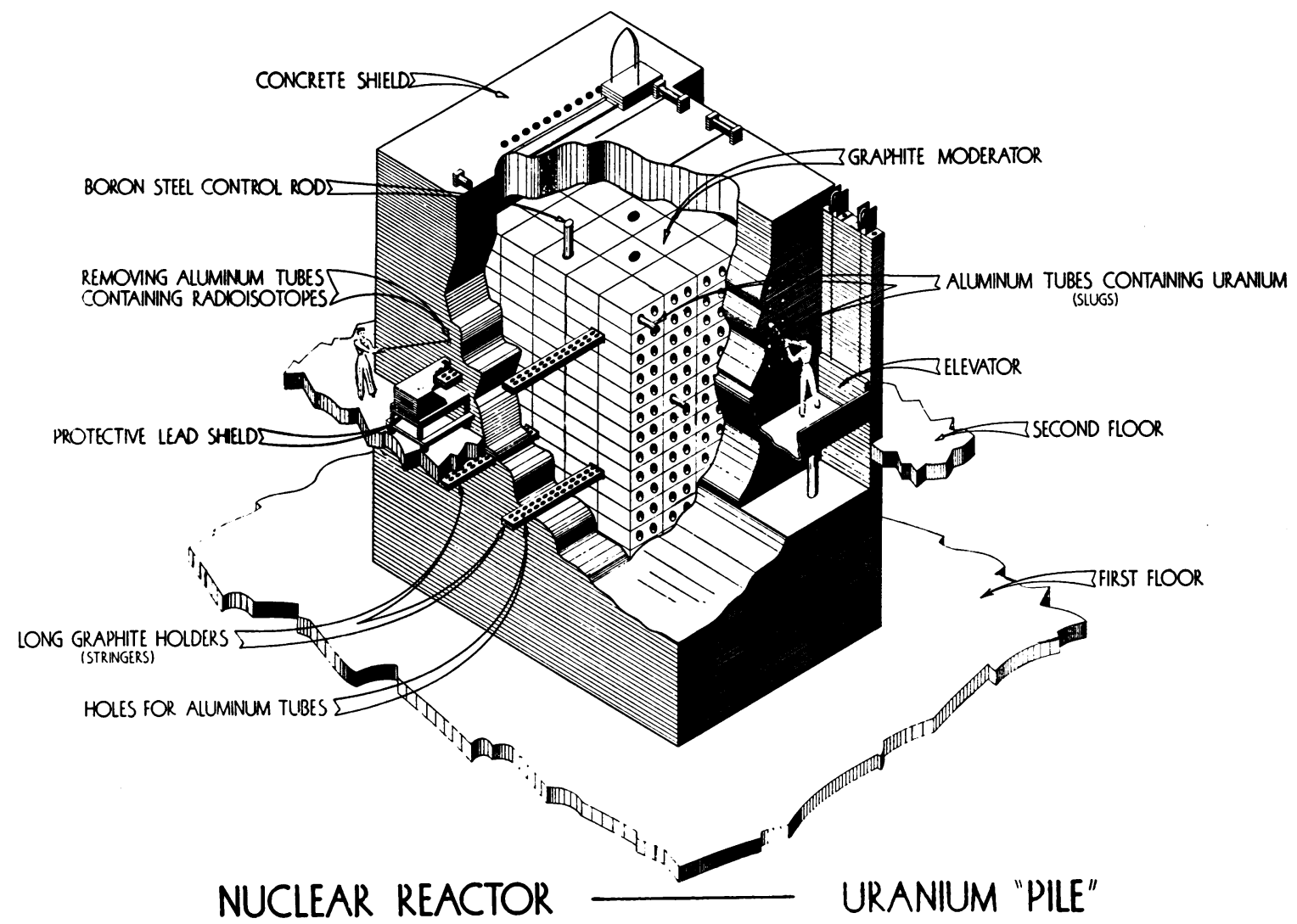

Fig. 3. Nuclear Reactor-Uranium Pile

The functional parts of the nuclear reactor are the fissionable material (uranium), the moderator material (graphite) and the control rods (boron steel). The uranium slugs are inserted into the pile from the right face (where the elevator is located). They are discharged at the opposite side of the pile into a channel located between the graphite and concrete. The slugs drop into water and are later chemically treated for removal of the fission products. The radioisotopes formed by the neutron bombardment of stable isotopes are irradiated in graphite holders or stringers as indicated on the left. The control rods are lowered into the pile to retard or stop the chain reaction. The thick concrete wall surrounding the reactive portion of the pile serves as protection for operating personnel.

Most of these shipments have been for medical and clinical investigations.

Today, pile production of radioisotopes has been stepped up to a point where these valuable research tools are available to all qualified investigators. In fact, the situation existent before the advent of pile production, when there was not an adequate supply of radioisotopes to meet the demands of qualified investigators, has been reversed. The present limitation to wider use of radiomaterials is the lack of personnel having the special training and facilities to use them safely. And yet, the number of qualified investigators has been multiplied several times above the prewar level.

\section{AVAILABILITY}

Radioisotopes are potentially hazardous to health and for this reason special facilities and techniques are required in handling and using them. The Atomic Energy Commission's primary concern in making radioisotopes available is that they will be used safely. To insure this provision, the Commission has set up certain criteria for the allocation of radioactive isotopes. The Commission has also procured the services of an Advisory Committee on Isotope Distribution to assist in the allocation of these valuable and yet potentially hazardous materials. 
The Subcommittee on Human Applications, a subcommittee of the main Advisory Committee, reviews all radioisotope requests proposing use of radiomaterials in humans. This subcommittee has outlined the following general aims governing the distribution of radioactive materials for medical purposes: (1) to make radioisotopes available for approved clinical investigations and for diagnostic and therapeutic purposes where they are needed and can be handled properly and safely; (2) to protect, as far as possible, patients from indiscriminate use of radioisotopes; and (3) to insure that adequate facilities are available for those planning to use the material.

In carrying out these very worthwhile aims the Commission is faced with two opposing purposes: first, to make these materials widely available and of maximum usefulness to medical research and second, to limit distribution sufficiently to guarantee their safe utilization. To achieve the desired balance between these two somewhat contradictory purposes, the Subcommittee on Human Applications has formulated criteria for allocation.

\section{Criteria of Allocation}

These criteria have proved satisfactory for the administration of the distribution program. Their merit is clearly evident from enumeration of the requirements: (1) physicians using radioactive material must be associated with a medical research institution, hospital, clinic or other organization having adequate facilities for handling the material; (2) such facilities must provide clinical care of the patient and adequate means for assaying, safely handling and disposing of the radioactive material; (3) the organization requesting the radioisotopes must be in good standing with the local medical society; (4) it is also strongly recommended that the requesting institution appoint a clinical "Isotope Committee" to evaluate all proposals for clinical use of the material within the institution; (5) the scientifically trained individual who will use or directly supervise the use of radioactive material must be an accredited physician in good standing with the local medical society; (6) also, the physician must have had previous clinical experience with radiation or radioactive materials or be directly collaborating with an individual possessing such training and experience.
In connection with the above recommendation the Subcommittee on Human Applications has recently adopted the policy of not approving a radioisotope request unless it bears a written statement of approval from the local clinical isotope committee. It is suggested that this local committee includes: (a) a physician trained in internal medicine; $(b)$ a physician trained in hematology; (c) a person experienced in assay of radiomaterials and protection of personnel against ionizing radiations; and, whenever possible, $(d)$ a qualified physicist, and $(e)$ a therapeutic radiologist. If a physicist and radiologist are not staff members of the requesting institution, they should be available in a consulting capacity.

Information indicating that the requesting institution meets these qualifications together with more detailed information concerning the proposed use of the material and proposed health safety measures is forwarded by the applicant in his formal request. Following review and approval of the request by the Subcommittee on Human Applications, the applicant is authorized to procure the material. Subsequent arrangements concerning purchasing the material and scheduling of shipment are made between the applicant and the supplier.

If the radioactive material is not intended for use in humans, the request is not reviewed by the Subcommittee on Human Applications. For animal studies, as well as for all other types of radioisotope investigations, prior reference of all requests to a local isotope committee is strongly recommended. In institutions where clinical and nonclinical investigations are carried on by different groups, a separate isotope committee may be established for the control and coordination of the use of isotopes in the non-clinical studies. Review by a local committee assures the Commission that the proposed program is considered to be both feasible and safe from the standpoint of the institution.

\section{Basic and Special Products}

When the Atomic Energy Commission inaugurated its distribution program, it focussed its attention primarily on producing a wide variety of isotopes rather than on producing a wide variety of compounds of a small number of isotopes. Indeed, early production was aimed at making 
the largest number of isotopes available in the simplest and most generally adaptable forms. By thus placing emphasis on producing simple basic forms for each isotope it has been possible to make these "basic products" available cheaply and on a large scale.

In many instances, the radioisotope users can conveniently use the materials in the simple basic forms as shipped. Radioiodine (I 131) and radiophosphorus ( $P$ 32), for instance, are often clinically administered as solutions of sodium iodine and disodium hydrogen phosphate which are the chemical forms in which the materials are shipped from the production site. In other cases, however, users may require a modified form or even a specially synthesized compound containing the isotope.

These specially synthesized compounds containing radioisotopes, "labeled" compounds, are extremely important to clinical and medical investigations because they permit tracer studies to be made of the molecules and parts of molecules which enter into biochemical and physiological processes. The element carbon, for instance, is one of the body's major constituents, although, of course, not in its elemental form. Indeed, it is always found in some complex molecule such as an amino acid or sugar. Therefore, in using radioactive carbon 14 to study a certain body compound it is necessary first to incorporate the radioisotope in the specific compound to be studied. These labeled or tagged compounds can be classed as "special products." They may vary from simply the modification of the material shipped, say from the carbonate into sulfate or sulfide, to the complicated synthesis of complex organic molecules.

The Commission has not undertaken an extensive program within its own facilities to prepare and make available these special products. It would not only be difficult for the Commission to maintain facilities and personnel to meet all the special needs of off-Commission users, but this is an area of endeavor which is free of security considerations and one which logically should be handled by private enterprise. Limited quantities of labeled compounds which are obtained as byproducts of Commission research activities are made available to off-Commission laboratories when prepared in excess of Commission needs.
The Commission has, however, encouraged commercial firms to engage in the preparation of special products. At the present time three organizations outside Commission laboratories are preparing and distributing isotope-labeled compounds and several other organizations are considering arrangements for initiating similar programs. There are over 50 such compounds now available and 35 additional compounds are scheduled to become available within the next six months. Although most of these compounds are labeled with radiocarbon ( $\mathrm{C} 14$ ), some compounds have been labeled with radioisotopes of sulfur, iodine, gold, zinc and cobalt.

Even though allocation of these materials is necessarily controlled by the Commission, all other arrangements relative to their procurement are made by the supplier and the radioisotope user. Also in its effect to encourage preparation and sale of isotope-labeled compounds by private enterprise, the Commission has adopted the policy of discontinuing distribution of any compound which becomes available from an outside source.

Besides its efforts to encourage wider use of radioisotopes through cooperating with private enterprise in programs such as mentioned above and through its support of training and educational programs, the Commission has also stepped up and amplified its own program of producing basic radioisotope products. New facilities for producing and processing these products are being designed and constructed. The Commission has made an effort to encourage the growth of medical and biological research and evidence of this was noted last April when it was announced that radioactive phosphorus ( $\mathrm{P} 32$ ), radioactive iodine (I 131), and radioactive sodium ( $\mathrm{Na} 24$ ) would be made available free of production costs for use in therapy, diagnosis, and research in cancer and allied diseases. More recently a further announcement was made stating that this program would not only be continued but expanded to include all isotopes normally available under its isotope distribution program providing the material is used in cancer research. More specifically the supplementary extension of the program provides for allocation of materials free of production cost for use in (1) cancer investigations involving animal 
subjects, (2) research programs studying basic cellular metabolism of cancerous cells, and (3) experimental programs designed to evaluate the uses of radioactive materials in the therapy and diagnosis of cancer.

\section{CONCLUSION}

In pointing out the present widespread availability of radioisotopes as a research tool, it must also be noted that this is based on much more than merely the increased availability of isotopes per se. Not only are radioisotopes available in amounts many times the prewar quantities, but they are also readily available as a much greater variety of radioelements. Nearly all the biologically useful radioisotopes, including such important ones as radiohydrogen (tritium, $\mathrm{H} 3$ ), radiocarbon ( $\mathrm{C}$ 14), radiophosphorus (P 32), radiosulfur (S 35), radiocalcium ( $\mathrm{Ca} 45$ ), radioiodine (I 131), etc., are produced routinely and are available continuously.

Further, a stock of isotope-labeled compounds is available for the first time. Many complex organic molecules have been labeled or tagged with iso- topes and many more will become available in the near future. Heretofore, tracer studies with isotopes were usually limited to following only the radioelement or its simple compounds. The availability of a wide variety of labeled organic and biochemical compounds will certainly promote much more extensive and diversified utilization of isotopes in biology and medicine.

Increased availability of materials and instruments has led recently to a much greater number of hospitals, clinics and other institutions using radioactive materials in routine diagnosis and therapy procedures and in research investigations. Before the war, for instance, little more than a dozen institutions were routinely using radiophosphorus (P 32) and radioiodine (I 131) in diagnosis and therapy. Today 80 different institutions are using either or both of these isotopes in this type of clinical work.

It seems quite certain that with the background at hand and the impetus now gained, future research with isotopes, especially isotope-labeled compounds, will lead to valuable new routine uses of radioisotopes in diagnosis and therapy. 